\title{
Deposition of ordered two-phase materials using microfluidic print nozzles with acoustic focusing
}

\author{
Rachel R. Collino ${ }^{\mathrm{a}, \mathrm{b}}$, Tyler R. Ray ${ }^{\mathrm{a}}$, Rachel C. Fleming ${ }^{\mathrm{c}}$, James D. Cornell ${ }^{\mathrm{d}}$, Brett G. \\ Compton ${ }^{\mathrm{e}}$, Matthew R. Begley ${ }^{\mathrm{a}, \mathrm{b}}$ \\ ${ }^{a}$ Department of Mechanical Engineering, University of California, Santa Barbara, CA 93106 \\ ${ }^{b}$ Materials Department, University of California, Santa Barbara, CA 93106 \\ ${ }^{c}$ Chemistry Department and Biochemistry Program, University of California, Santa Barbara, CA 93106 \\ ${ }^{d}$ Department of Electrical Engineering, University of California, Santa Barbara, CA 93106 \\ ${ }^{e}$ Mechanical, Aerospace and Biomedical Engineering Department, University of Tennessee, Knoxville, TN \\ 37996
}

\begin{abstract}
The deposition of multiphase materials with microstructural control would enable new fabrication modalities, such as spatial variation in composition and the integration of microstructure and structural features. In this work, acousticallyexcited microfluidic print nozzles were used to tailor the microstructure of printed composite filaments consisting of $\mathrm{SiC}$ fibers, solid $\mathrm{BaTiO}_{3}$ spheres, or hollow $\mathrm{SiO}_{2}$ spheres in an epoxy matrix. The results demonstrate that acoustic focusing is a promising technique to control microparticles and deposit two-phase ordered structures using a single nozzle. In addition to tuning the microstructure within a single print line, the overall concentration of particles can be increased by focusing the particle stream in a branched geometry, enabling the deposition of material with higher particle volume fractions than the initial ink composition. We show that even modest volume fractions of acoustically-focused and concentrated $\mathrm{SiC}$ fibers can produce printed composite filaments with unprecedented control over microstructural ordering that exhibit strengths rivaling polymer-matrix composites with higher volume fractions of stiffer fibers.
\end{abstract}

Keywords: additive manufacturing, 3D printing, microfluidics, acoustic waves

\section{Introduction}

There is significant motivation to develop direct deposition techniques for composites, particularly those capable of producing ordered phases. Such techniques would unleash the true power of additive manufacturing, in that the printing process could directly couple spatial variations in composition (including both volume fraction and phase orientation) with structural features of components. Notable examples include functionally graded soft robots[1], monolithic[2,3] and lattice composite materials with strong, stiff reinforcements aligned with cell walls[4], 
and dense lines of conductive particles for electrical interconnects[5]. In such applications, the ability to control anisotropic properties (mechanical, electrical, thermal, and optical) offers considerable opportunities for new levels of multifunctional performance: for example, the exploitation of percolation limits to introduce electro-mechanical coupling.

Arguably, there are two central strategies for the fabrication of ordered composites consisting of disparate phases. The first involves deposition of multiple monolithic inks with high spatial resolution, in which ordering is achieved through direct patterning. For conventional inkjet printing, minimum droplet sizes and droplet spreading limit the final resolution of phase ordering[6]. A promising advance in inkjet printing is electrohydrodynamic printing (e-jet), which improves spatial resolution[7]. E-jet has been used to successfully deposit microspheres that form arrays post-deposition[8] as well as nanowires that order in response to an applied electrical field[9]. In a similar vein, magnetic-field-assisted 3D printing has also been demonstrated for the deposition of ordered nanowire patterns[10] and micropillars[11]. These require specific substrate conditions, such as predetermined grid arrays[10] or wetting properties[11]. Advances in inkjet printing create obvious opportunities for multi-material printing, as has been demonstrated via ejet in a configuration with multiple printheads[12]. However, such methods raise significant questions regarding the bandwidth of suitable inks (having the requisite electrical and/or magnetic properties), as well as the scalability with regard to component size.

An alternative strategy to generate ordered composites (currently less developed than dual-nozzle strategies) involves the development of multi-component inks, in which particles are suspended in a fluid that ultimately forms the composite matrix. Extrusion techniques have been developed to directly deposit particleladen fluids that align in response to electric fields[13,14] or hydrodynamic effects, as demonstrated for glass in epoxy[3] and carbon fibers in acrylonitrile-butadienestyrene (ABS)[15] and epoxy[2,4]. Such methods offer the important advantage of being scalable to large components. However, extrusion-based techniques alone typically require careful control of the rheology of the base fluid, and the particle alignment and packing is limited by hydrodynamics of the process[3,16].

In this work, the advantages of field-assisted deposition are combined with those of extrusion-based processes through the use of acoustic fields, which are used to drive particle alignment and packing in particle-laden inks. In contrast to electromagnetic forces, the generation of acoustic forces is relatively material agnostic, requiring no specific material properties other than density or compressibility differences between particle and matrix (as outlined below). Large forces, sufficient to transport particles over millimeter distances on the order of seconds, can be generated via standing pressure waves induced with inexpensive piezoelectric actuators. As such, acoustic-field-assisted deposition is highly complementary to other techniques, and is compatible with previous advances in chemical self- 


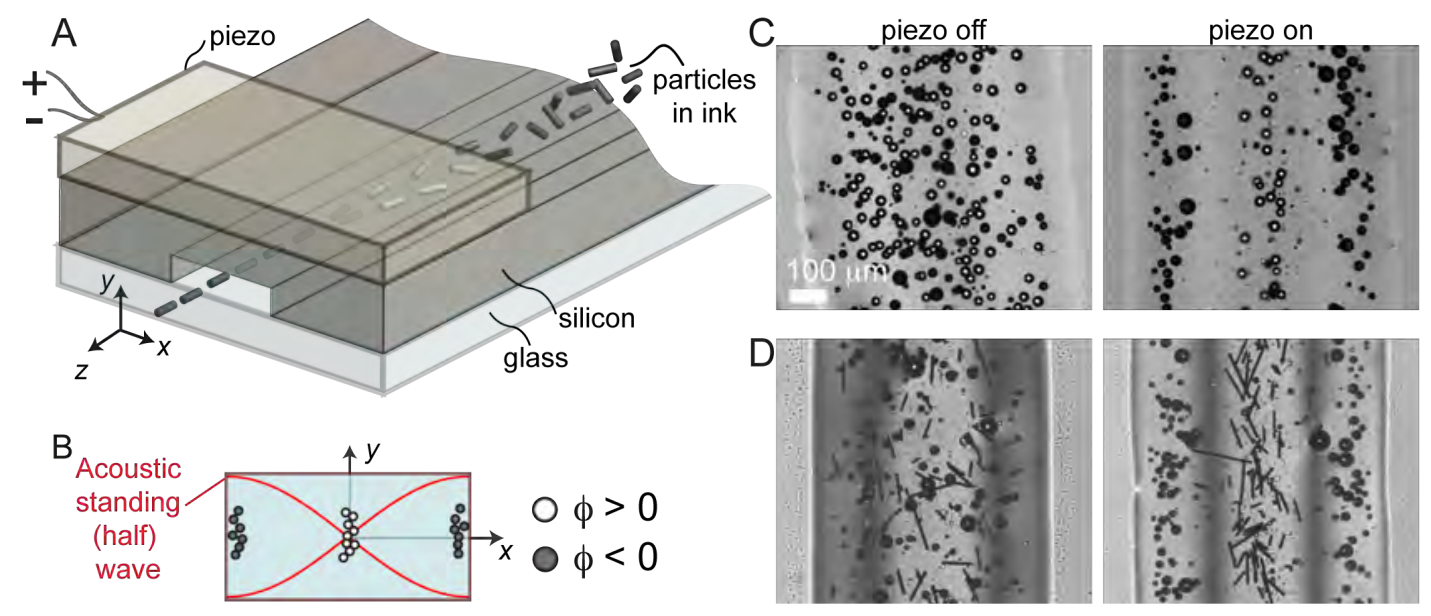

Figure 1: (A) Print nozzle channel geometry and coordinate system. Inks comprised of particles suspended in a fluid base are delivered to silicon/glass devices. A coupled piezoelectric element is excited at a frequency corresponding to a half-standing-wave in pressure in the fluid. (B) Particles with positive acoustic contrast factor $\phi$ focus to the pressure node; particles with negative $\phi$ focus to anti-nodes. (C) Examples of particle separations (induced in the microchannel and retained in printed lines) for solid and hollow spheres as well as (D) SiC fibers and hollow spheres.

assembly or hydrodynamic focusing.

Acoustically-assisted deposition is suitable for the manufacture of materials that may be difficult to achieve with discrete drop-on-demand technologies, such as those requiring aligned, high-aspect ratio fibers. In addition, acoustic focusing is gentle enough for the manipulation of fragile constituents; for example, classical applications of acoustophoresis in microchannels include cell sorting [17, 18] and filtration for biological and medical analyses[19, 20]. In static structures, acoustic waves have been used to assemble microgels[21] as a platform for various biotechnological applications. Thus, the pairing of acoustic focusing with deposition offers powerful opportunities for the manufacture of both structural materials as well as mechanically and thermally-sensitive systems[22].

In this work, preferential particle alignment and packing is demonstrated using acoustic-field-assisted deposition, with examples of multi-phase microstructure deposition achieved through tuning of acoustic excitation parameters and deposition conditions. We show that branched nozzle geometries can be used to focus particles into a central print pathway to deliver composite filaments with higher volume fractions than the starting ink. Indeed, we demonstrate that acoustic focusing can be used to print ink compositions that would otherwise lead to jamming. To illustrate potential performance gains, the mechanical properties of printed SiC- 
epoxy filaments have been directly measured. A major component of this work is the development of an ink with sufficient elasticity to retain structure upon deposition while also balancing time scales for acoustic focusing to enable reasonable print speeds (on the order of several millimeters per second). This is an important new advance, since achieving assembly concurrent with transport in a viscoelastic medium is distinct from static patterning in acoustic fields[23, 24, 25, 26] which requires parts to be subsequently cut from molds[26].

The acoustic field-assisted deposition process described here is governed by the scaling of acoustic forces generated on suspended particles in standing pressure waves[19, 27, 28, 29, 30, 31, 32, 33]. There are two types of forces generated by the standing pressure wave: the primary acoustic radiation force (PARF), which acts to focus the particles to the center of the channel, and secondary acoustic scattering forces, which act to repel particles in close proximity in the direction of wave propagation. In the present experiments, the primary forces are dominant; we summarize the scaling equations for these forces to provide insight into the types of particles that can be focused. A companion paper explores the potential role of scattering forces with regard to printing[34].

The coordinate system and geometry of the channel are shown schematically in Figure 1, where the standing wave propagation direction $x$ is in the channel width dimension, with the ordinate at the channel center. The primary acoustic radiation force acts in this direction to drive particles to nodes or anti-nodes of pressure according the contrast factor $\phi$ (for positive and negative values of $\phi$, respectively), as shown in Fig. 1A,B. The normalized, time-averaged value of the primary acoustic radiation force $\bar{F}_{p}$ is:

$$
\bar{F}_{p} \equiv \frac{F_{p}}{F_{o}}=-\sin (2 \pi \bar{x}) ; \quad F_{o}=\frac{\pi^{2} a^{3} \beta_{f} p_{o}^{2} \phi}{6 W} ; \quad \phi=\frac{5 \rho_{p}-2 \rho_{f}}{2 \rho_{p}+\rho_{f}}-\frac{\beta_{p}}{\beta_{f}}
$$

where $F_{0}$ is the focusing force amplitude, $\bar{x}=x / \mathrm{W}$ is the normalized particle position, $p_{o}$ is the pressure, $W$ is the channel width, $a$ is the particle radius, $\rho$ is density, and $\beta$ is compressibility, where the subscripts $f$ and $p$ refer to the fluid and particle, respectively.

A demonstration of the effect of the contrast factor in ordering deposited structures is shown in Figs. 1C,D: the images on the left illustrate that, in the absence of piezoelectric excitation, ink lines are deposited without any apparent particle ordering. Results are shown for solid $\mathrm{BaTiO}_{3}$ spheres, hollow glass spheres, and $\mathrm{SiC}$ fibers. When the piezoelectric is tuned to induce a standing half-wave in pressure (Fig. 1C,D - right), the less-dense hollow spheres are driven to the pressure anti-nodes near the channel walls whereas the solid spheres and fibers focus to the center pressure node, resulting in print lines with clearly delineated ordering. In both cases, steady-state particle separation is achieved in the channel within a few seconds, and these separations are retained in the printed lines as illustrated in Fig. 1C,D - right). 


\section{Materials and Methods}

\subsection{Device fabrication and printing}

Devices consisting of etched silicon channels ( $\sim 150 \mu \mathrm{m}$ deep) and glass capping layers were fabricated according to the procedures in Ref. [35]. For singlechannel studies, $1 \mathrm{~mm}$ thick, $20 \mathrm{~mm}$ diameter piezoelectric ceramics (Meggitt, PZ26 material) were coupled to devices with $\sim 12 \mathrm{~mm}$ long, $350 \mu \mathrm{m}$ wide channels and excited at $2.09 \mathrm{MHz}$. For branched channel studies, channel geometry consisted of $350 \mu \mathrm{m}$ wide, $\sim 12 \mathrm{~mm}$ long inlet channels, a $220 \mu \mathrm{m}$ wide, $16 \mathrm{~mm}$ long central outlet channel, and two $110 \mu \mathrm{m}$ wide, $2 \mathrm{~mm}$ long 'waste' channels. For single-stage focusing studies in branched channels, a $1 \mathrm{~mm}$ thick piezoelectric element (as described above) was positioned over the $350 \mu \mathrm{m}$ wide channel and excited at either $2.04 \mathrm{MHz}$ (for $\mathrm{SiC}$ Ink C) or $2.09 \mathrm{MHz}$ (all other inks); for twostage studies, a second piezoelectric element, $0.5 \mathrm{~mm}$ thick and $10 \mathrm{~mm}$ square, was placed over the outlet channel near the nozzle exit and excited at $3.32 \mathrm{MHz}$. In all cases, an ultrasonic coupling gel (PosiTector, DeFelsko Corp.) was used to attach piezoelectric elements to the silicon print nozzle surfaces.

Inks were delivered to acoustic nozzles using controlled pressure (Fluigent MFCS-EZ) and particle motion was monitored optically during deposition (Infinitube FM-200 coupled with a Point Grey camera, FL3-U3-32S2M-CS). For the monolithic-channel-deposited $\mathrm{SiC}$ fiber print lines shown in Fig. 3 and Fig. 6A, input pressures of 150, 80, and $50 \mathrm{kPa}$ were used for 0,16 , and $23 \mathrm{~V}$ focusing conditions, respectively, with stage speeds as indicated in Fig. 3. For sphere-filled inks in branched channels, deposition pressures and stage speeds were $120 \mathrm{kPa} / 1 \mathrm{~mm}$ $\mathrm{s}^{-1}, 160 \mathrm{kPa} / 5.6 \mathrm{~mm} \mathrm{~s}^{-1}$, and $180 \mathrm{kPa} / 5.6 \mathrm{~mm} \mathrm{~s}^{-1}$, respectively, for the control, first-stage, and second-stage deposition shown in Fig. 5B-D. For SiC-fiber inks in branched channels, pressures and speeds of $150 \mathrm{kPa} / 4.7 \mathrm{~mm} \mathrm{~s}^{-1}$ and $50 \mathrm{kPa} / 7.5$ $\mathrm{mm} \mathrm{s}^{-1}$ were used for unfocused and concentrated deposition as shown in Fig. 6B and $C$, respectively.

Excitation signals were supplied to piezoelectric elements as described in Ref. [35] via a waveform generator (Fluke 294) and amplifier (Mini-Circuits LZY-22+), and the voltage amplitude and frequency monitored via an ocscilloscope (Agilent DSO$X$ 2024A). In most cases, deposition of printed lines was achieved by positioning a stationary, level print nozzle above a moving substrate (plain glass slide) at a fixed angle $\left(\sim 35^{\circ}\right)$; print speed was controlled via a screw-driven linear stage (Zaber T-LSR150B). For microstructural modulation studies of $\mathrm{SiC}$ and $\mathrm{BaTiO}_{3}$ particles in epoxy, a ShopBot Desktop (ShopBot Inc.) was used to position the nozzle over a stationary slide at a similar angle, with driving pressure of 56 and $100 \mathrm{kPa}$, respectively, and a print head velocity of $1.3 \mathrm{~mm} \mathrm{~s}^{-1}$. Post-deposition, ink lines were cured on a $180^{\circ} \mathrm{C}$ hot plate for 90 minutes. For samples that were subsequently mechanically tested, lines were deposited on a layer of high-temperature fluoropolymer-coated tape (McMaster) attached to the glass slides and triangular 
tabs were molded (from the base ink) to the ends of the print lines for gripping in the tensile tester.

\subsection{Ink formulation and rheological characterization}

$\mathrm{SiC}$ fibers (SI-TUFF SF-7, a gift from Advanced Composite Materials) were sifted through 250,125, 63, and $20 \mu \mathrm{m}$ mesh screens to select particle lengths of $\sim 20-63 \mu \mathrm{m}$. Base inks consisted of 50:3:2.6 parts by weight of epoxy resin (MillerStephenson EPON 828):fumed silica (Evonik Aerosil R106):hardening agent (Basionics VSO3), mixed for three increments of 3 min each, allowing the mixture to cool to room temperature before each addition. Inks with particles were made by adding acetone and particles to the base ink and mixing for an additional $3 \mathrm{~min}$. In all cases, the ratio of base ink to acetone was 12:1 by weight except for two-stage $\mathrm{SiC}$ fiber ink tests and microstructural modulation tests with $\mathrm{SiC}$ fibers or $\mathrm{BaTiO}_{3}$ spheres, for which a ratio of 6:1 was used. It is assumed that all acetone evaporates in the deposition/curing process, so the final printed ink compositions (neglecting acetone) are given in Fig 2B. Particle densities, in $\mathrm{g} \mathrm{cc}^{-1}$ as reported by their respective manufacturers, are: 3.04 ( $\mathrm{SiC}$ fibers, $7 \mu \mathrm{m}$ diameter), $4.2\left(\mathrm{BaTiO}_{3}\right.$ solid spheres, $34.5 \mu \mathrm{m}$ diameter, Corpuscular), 0.6 (hollow glass spheres, $31 \mu \mathrm{m}$ diameter, Corpuscular). All mixing steps were performed in a planetary mixer (Thinky ARE-310) at $2000 \mathrm{rpm}$, and all nominal compositions determined with a microbalance (Mettler AE163). Rheological properties of inks were measured using a rheometer (LC-ARES, Rheometric Scientific) fitted with $50 \mathrm{~mm}$ diameter parallel plates with a gap of $0.5 \mathrm{~mm}$, conditioned for $1 \mathrm{~min}$ at $100 \mathrm{~s}^{-1}$ prior to testing.

\subsection{Mechanical testing and microstructural characterization:}

Tensile tests were performed in a screw-driven stage fitted with a $10 \mathrm{lb}$. load cell (Eaton 3108-01) with a crosshead speed of $0.2 \mathrm{~mm} \mathrm{~min}^{-1}$. Tensile test specimens consisted of $25 \mathrm{~mm}$ long printed lines with dogbone-style grips molded at the ends with the epoxy base ink. Typical gauge lengths were $10 \mathrm{~mm}$, with crosssectional area of approximately $0.125 \mathrm{~mm}^{2}$. Strain was measured using a laser extensometer (Electronic Instrument Research LE-01) at a sample rate of $20 \mathrm{~s}^{-1}$; plots represent a moving average of the data over $1 \mathrm{~s}$ intervals. Optical micrographs of printed lines in plan-view were obtained in Nikon TI-U Eclipse, Nikon Eclipse ME600, or Keyence VHX-5000 microscopes. An estimate of fiber vol\% in print lines for which the printed particle vol\% is increased (after passing through a single focusing stage and discarding excess base ink) was determined by manual area fraction measurements through successive focal planes in the Nikon TI-U Eclipse microscope. Cross-sectional images in Fig. 3B were obtained with a scanning laser confocal microscope (Olympus LEXT OLS4000) with polarized brightfield illumination and post-processed and analyzed in LEXT OLS software. Cross-sectional samples were prepared by fracturing printed lines and polishing with diamond lapping films (Allied High Tech), to a final grit size of $0.25 \mu \mathrm{m}$. All image analysis was performed using ImageJ software[36]. 
A

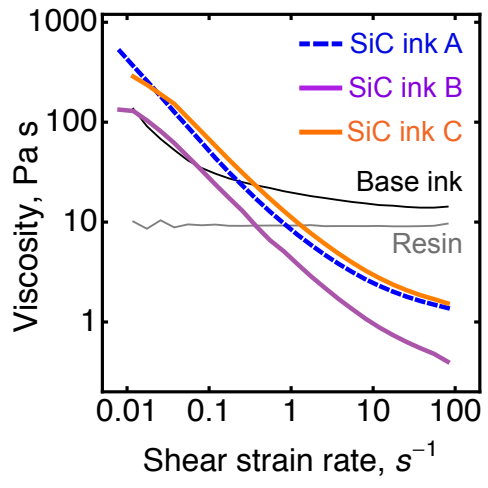

B

\begin{tabular}{|c|c|c|c|c|c|c|c|c|}
\hline \multirow[b]{2}{*}{ Ink Type } & Base ink & $\begin{array}{l}\text { e ink } \\
\text { o silica) }\end{array}$ & \multicolumn{2}{|c|}{$\mathrm{SiC}$} & \multicolumn{2}{|c|}{$\mathrm{BaTiO}_{3}$} & \multicolumn{2}{|c|}{$\mathrm{SiO}_{2}$} \\
\hline & $w t \%$ & vol\% & $w t \%$ & vol\% & $w t \%$ & vol\% & $\overline{w t} \%$ & vol\% \\
\hline $\mathrm{SiC} / \mathrm{A}, \mathrm{B}$ & 98.0 & 99.2 & 2.0 & 0.8 & & & & \\
\hline $\mathrm{SiC} / \mathrm{C}$ & 95.2 & 98.1 & 4.8 & 1.9 & & & & \\
\hline Spheres/Solid & 88.2 & 96.3 & & & 11.8 & 3.7 & & \\
\hline Spheres/Mixed & 86.7 & 92.8 & & & 11.6 & 3.5 & 1.7 & 3.7 \\
\hline $\mathrm{SiC} /$ Hollow & 96.8 & 95.7 & 1.3 & 0.5 & & & 1.9 & 3.8 \\
\hline
\end{tabular}

Figure 2: (A) Viscosity of the resin, base (without particles), and SiC particle laden inks. (B) Composition of the printed inks; it is assumed that all acetone (mixed in the feed ink with proportions given in Methods) evaporates post-deposition.

\section{Results}

\subsection{Ink development}

To develop inks suitable for both acoustic focusing and deposition, two major considerations are (i) the ability to focus particles at time-scales compatible with printing, and (ii) identification of an ink base with sufficient elasticity to retain the ordered structure post-deposition. In previous work, ordered assemblies were locked in a hydrogel matrix via photopolymerization[35]; for this work we select an epoxy-based matrix similar to that reported in Refs. [2,4] to allow for direct deposition of structurally-robust print lines. In the present case, fumed silica is chosen as a filler due to its miscibility in the epoxy resin (to avoid aggregation) and ability to impart elasticity to the base ink. The addition of silica and curing agent increases the viscosity of the epoxy resin and also introduces shear-thinning behavior as shown in Fig. 2A. Both the fraction of fibers (Fig. 2B) as well as the fraction of solvent (acetone) used in the ink was varied as described in Methods, and it was found that the fiber fractions studied here had little effect on the viscosity of the ink, which was more strongly a function of the amount of acetone. This is apparent from the data presented for $\mathrm{SiC}$ Inks $\mathrm{A}$ and $\mathrm{B}$ in Fig. 2A, in which the amount of acetone is increased in the latter. When the particle loading is increased from 0.8 to $1.9 \mathrm{vol} \%$ (between SiC Inks A and C) there is very little difference in viscoelastic behavior.

\subsection{Printed composite filaments using single channels}

To investigate the effect of focusing and deposition conditions on the microstructure of printed filaments with embedded $\mathrm{SiC}$ fibers ( $\mathrm{SiC}$ Ink $\mathrm{A}$ ), the excitation voltage and stage speed were varied and the resulting microstructures examined in both plan-view and cross-section. Figure 3A is a schematic of the single-channel 


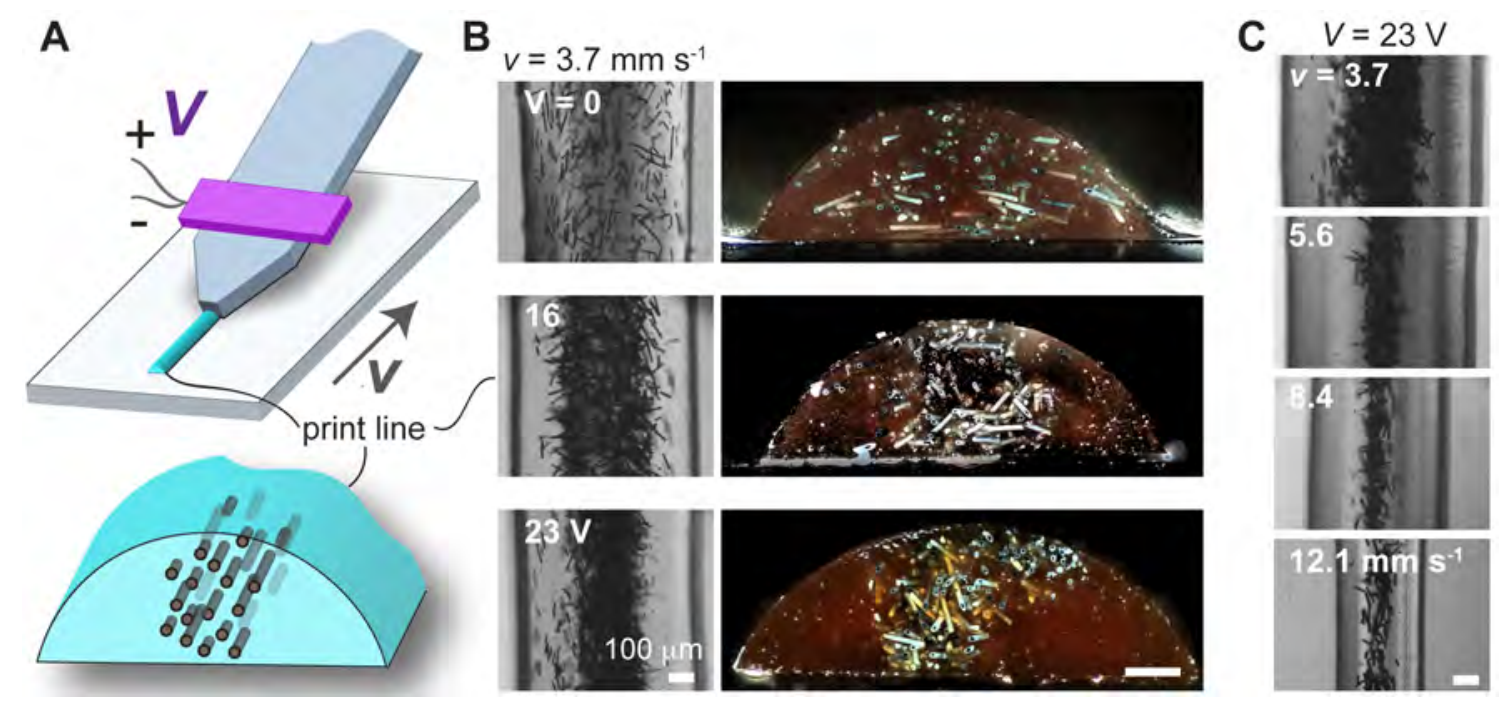

Figure 3: (A) Schematic of a microfluidic print nozzle with a coupled piezoelectric actuator driven at a peak-to-peak voltage $V$, with deposition speed controlled by the substrate velocity $v$. (B) Print line microstructures for a constant deposition speed of $3.7 \mathrm{~mm} \mathrm{~s}^{-1}$ and increasing excitation voltage of 0,16 , and $23 \mathrm{~V}$ in plan view (left) and cross-section (right). (C) Plan-view micrographs of print lines for the highest excitation voltage studied with deposition speed varied from 3.7 to $12.1 \mathrm{~mm}$ $\mathrm{s}^{-1}$. An ink with $0.8 \mathrm{vol} \% \mathrm{SiC}$ was used in all cases.

nozzle geometry; in all cases, the print nozzle is stationary and the print substrate moves relative to the printhead. The effect of excitation voltage on microstructure is shown in Fig. 3B for 0, 16, and $23 \mathrm{~V}$ (peak-to-peak, in all cases). For the control condition $(0 \mathrm{~V})$, no fiber focusing is apparent, and cross-sectional imaging reveals a distribution of fiber orientations; as the voltage is increased, deposited fibers focus to a smaller cross-section within the printed line.

To determine the effect of the relative velocity of the ink and printing substrate, for a given particle transport velocity within the channel, inks were driven at the same constant pressure as in Fig. 3B and at the maximum focusing voltage, with stage speeds varying from 3.7 to $12.1 \mathrm{~mm} \mathrm{~s}^{-1}$, as shown in Fig. 3C. As such, the particles spend the same amount of time in the focusing zone in the nozzle; print morphologies are thus a function of bead drawing effects at the exit. Tight particle focusing is retained at all but the highest print speeds where the ink ejection speed approximately matches that of the print substrate. Although ink ejection speed is not measured directly here, at higher stage velocities, print lines become discontinuous.

Critically, acoustic focusing enables control of the microstructure of the deposited line 'on the fly' by modulating acoustic excitation parameters, as demonstrated in Fig. 4 for $\mathrm{SiC}$ fibers in epoxy. In this case, when the excitation frequency is tuned to induce focusing ('focus on'), particles collapse to the center of the print line. The focusing transition occurs quickly enough to tune the microstruc- 


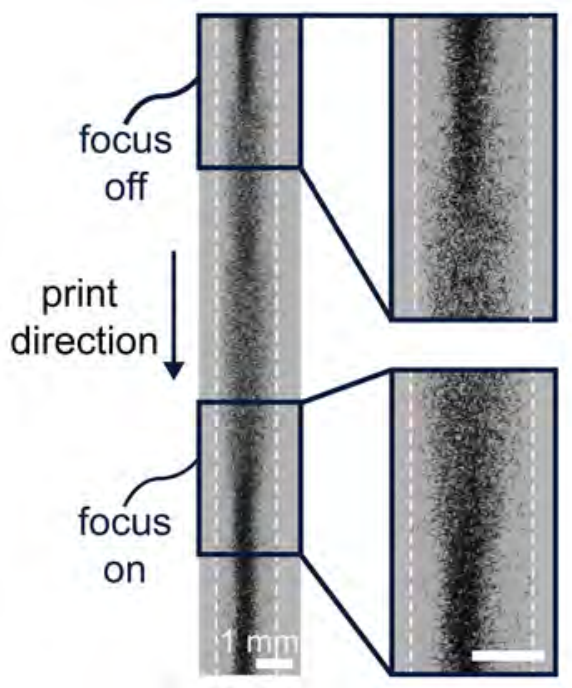

Figure 4: Printed line with continuously varied microstructure. The focusing frequency is tuned to induce focusing of $\mathrm{SiC}$ fibers in epoxy; when the frequency is tuned away from resonance ("focus off"), the fibers spread to the width of the deposited line. Dashed lines indicate the edges of the print line, which was deposited at $1 \mathrm{~mm} \mathrm{~s}^{-1}$.

ture from densely packed to distributed fibers within approximately 1-3 filament widths. When the excitation frequency is changed ('focus off'), particles spread nearly uniformly throughout the print line. The transition between focused and de-focused microstructures, and vice versa, is roughly symmetric (although flow conditions and particle geometry may affect this symmetry); this transition is influenced by the shape of the focus zone in the $(x, z)$-plane and the flow speed in the channel. While focusing time scales in the direction of the standing wave will be dominated by the primary acoustic radiation force as discussed above, defocusing time scales are more dependent on hydrodynamics. A companion paper describing the relationships between acoustic excitation, channel dimensions and focus zone size provides some insight as to the limits of how the microstructure can be varied axially within a given print line[34]. An additional example with $\mathrm{BaTiO}_{3}$ spheres is presented in Supplemental Figure 1.

\subsection{Printed composite filaments using branched channels and two-stage focusing}

In single channel print nozzles, focusing produces a 'sheath' of matrix surrounding the focused particles (as seen in Fig. 3). In some applications (such as structural composites), it would be desirable to remove this sheath prior to ejection. This can be accomplished with a branched channel, such as that shown in Fig. 5A. Figs. 5B-D illustrate that varying excitation conditions (top) can be used to alter print line composition and structure, as shown in plan-view (middle) and cross-sectional (bottom) micrographs of print lines consisting of $\mathrm{BaTiO}_{3}$ spheres in epoxy. With no piezoelectric excitation (Fig. 5B), particles flow through the central 
outlet as well as the two side branches; printing occurs only through the central outlet, the excess "flash" can either be discarded or recycled. In Fig. 5C, a piezoelectric is coupled to the inlet channel to produce a single focusing node which we refer to as 'first-stage focusing.' This leads to printed volume fractions of particles that are higher than in the starting ink, which enables new printing paradigms: focusing can be used to eliminate jamming associated with transport of higher particle concentrations. Particles lose focusing in the outlet channel to produce a concentrated, but random microstructure.

Since flow through the constriction at the channel branch can disrupt ordering, the strategy of using a second focusing stage was also explored as shown in Fig. 5D; the focusing frequency of the narrow output channel is different from that of the upstream channel, and as such, both focusing stages can be operated concurrently. In this case, the particles are re-focused and deposit in a dense centralized array within the print line.

As discussed above, when coupled with extrusion-based techniques, acoustic focusing is well-suited to the deposition of high-aspect ratio particles such as fibers, for structural composite applications. As such, we explored the parameters affecting the printability and microstructure of $\mathrm{SiC}$-fiber-filled epoxy inks in both single and branched channels as shown in Fig. 6. In Fig. 6A, SiC fibers are focused in a monolithic channel as shown schematically (top) and in a micrograph of the print line (bottom). Figs. 6B and C illustrate printing from branched channels without excitation (control) and with first-stage focusing, respectively. Secondstage focusing was not pursued in the present case for fiber composites; instead, we address the relative merits of increasing particle volume fraction via first-stage focusing versus additional densification toward the center of the print line. The latter does not necessarily improve mechanical properties, as discussed in the following section.

\subsection{Mechanical properties of printed composite filaments}

For the fabrication of polymer matrix composites with improved structural properties, elementary composite theory indicates that significant gains in mechanical properties can be achieved with even modest increases in the volume fraction of stiff fibers in a polymeric matrix[37]. Figure 7A shows representative tensile test results for base ink (with no particles) and inks with varying particle distribution (focused vs. unfocused) and vol\% of SiC fibers. Figure 7B presents the mean and standard deviation of Young's modulus and tensile strength for at least five samples in each case. The base ink (resin with fumed silica filler) exhibits appreciable ductility and moderate strength and Young's modulus. The addition of $0.8 \mathrm{vol} \% \mathrm{SiC}$ decreases ductility, and leads to a slight increase in modulus (in the unfocused case). Overall, the largest effects on mechanical properties are seen in lines printed in a two-stage device (as shown in Fig. 6C). These 'concentrated' specimens (estimated at $\sim 1.3 \mathrm{vol} \% \mathrm{SiC}$ ) exhibit moderately higher strength than 

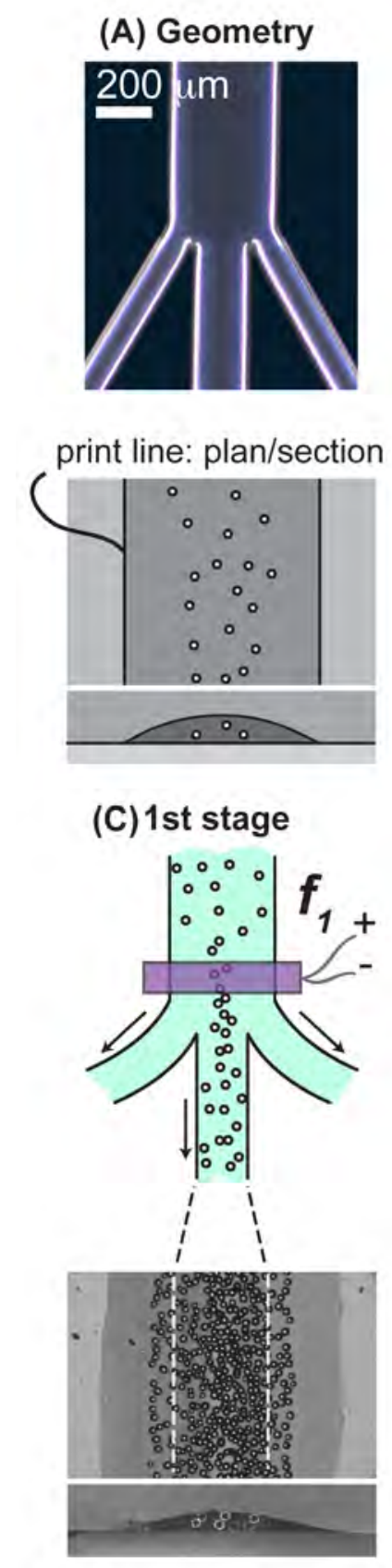

(B) Control

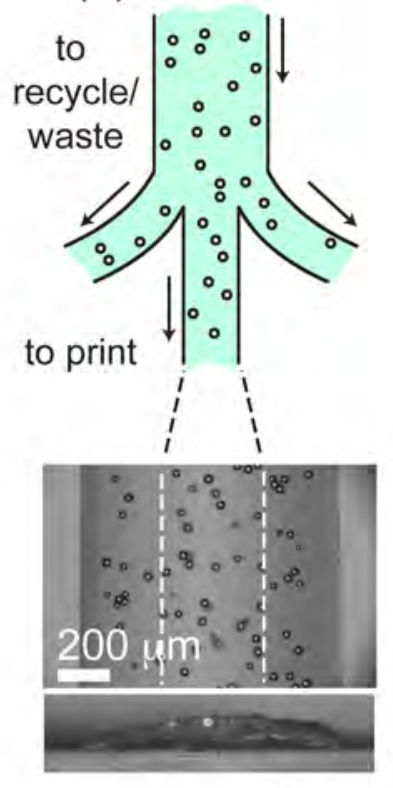

(D) +2nd stage

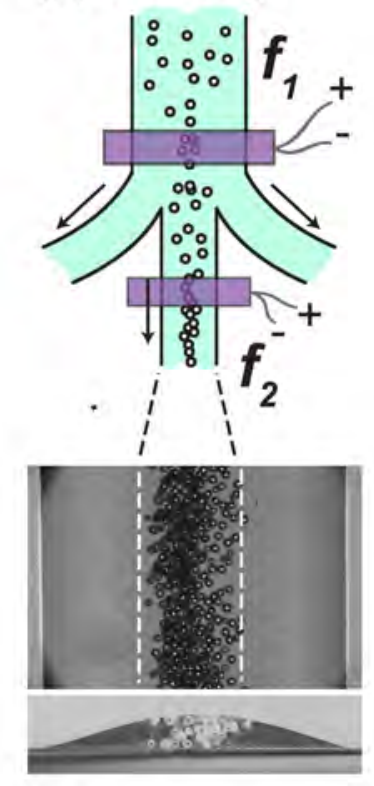

Figure 5: A) Micrograph of a branched, two-stage microfluidic channel (top) used to deposit print lines consisting of $\mathrm{BaTiO}_{3}$ spheres in epoxy with varying microstructure, shown schematically in plan-view (middle) and cross-section (bottom). (B) In the control condition with no piezo excitation at either stage, particles exhibit no apparent ordering within the print line. (C) A piezo coupled to the first stage (wider channel) is excited at a frequency $f_{1}$ to induce focusing of spheres to the center, concentrating the particles to the central outlet (printing) channel and peeling off excess matrix, to yield a print line with a higher volume fraction of disordered spheres. (D) A second piezo is coupled to the print outlet channel and tuned to its half-wave frequency $f_{2}$ to induce a second stage of ordering, focusing the concentrated particles to the center of the print line. The dashed lines indicate the dimensions of the output channel on the device. 
the single-channel focused specimens with $1.9 \mathrm{vol} \% \mathrm{SiC}$.

\section{Discussion}

Analysis of particle speeds during typical deposition conditions indicate a local shear strain rate on the order of $0.1 \mathrm{~s}^{-1}$ near the nozzle exit; thus, as inks are ejected and come to rest, the local viscosity increases by an order of magnitude or more, as suggested by Fig. 2A, allowing for the retention of ordered microstructures. While the deposition speeds reported here (several $\mathrm{mm} \mathrm{s}^{-1}$ ) are comparable with current consumer 3D printing technologies, an obvious goal is the increase of print speed and therefore effective build rates. While shear-thinning behavior is not necessary for the acoustic field-assisted deposition shown here, such inks are promising for the additional level of control of local viscosity: as build speed is scaled up, local viscosity decreases, which is advantageous for focusing.

However, a competing consideration is that as particle speeds increase, either the focusing or the transport time/distance must be scaled such that particles can reach the desired focusing position during transport through nozzles. A further complication is the effect of viscosity of the medium on particle behavior in standing waves, as the vast majority of acoustophoresis studies in microchannels focus on suspensions in relatively inviscid fluids. By contrast, most technologically relevant printable inks such as the polymer-based ones discussed here will possess viscoelastic properties; a more detailed treatment of the effect of viscosity and print parameters on particle behavior is treated in a companion paper[34].

In addition, effective particle flow speeds in an acoustically-excited print nozzle will result from both pressure-driven flow as well as acoustic-streaming-induced flow. Experimental[38] and theoretical[39] studies have shown that acoustic streaming becomes more pronounced for smaller particles[40] in viscous fluids; a balance of these flow contributions as a function of the acoustic energy density will be necessary for the precise control of print speeds. This is both an advantage (bulk streaming can be exploited to reduce printing pressures) and a disadvantage (pressures may have to be modulated in concert with acoustic excitation to achieve a desired flow rate).

The ability to couple localized piezoelectric excitation to different channel geometries creates the opportunity for versatile print modalities enabled by acoustic focusing. When focusing is induced in the 'exit' stage as in the single-channel nozzle (Figs. 3,6A) and the branched nozzle (Fig. 5D), a densely-packed array of particles within a sheath of matrix is produced. In some applications, such as the printing of percolating networks of conducting particles, this printing scheme could enable the fabrication of insulated conductive networks with a single print line. An intriguing possibility is the use of acoustic tuning to modulate microstructures near percolation thresholds, which would create composites with strong coupling between mechanical deformation and electrical performance. In these appli- 


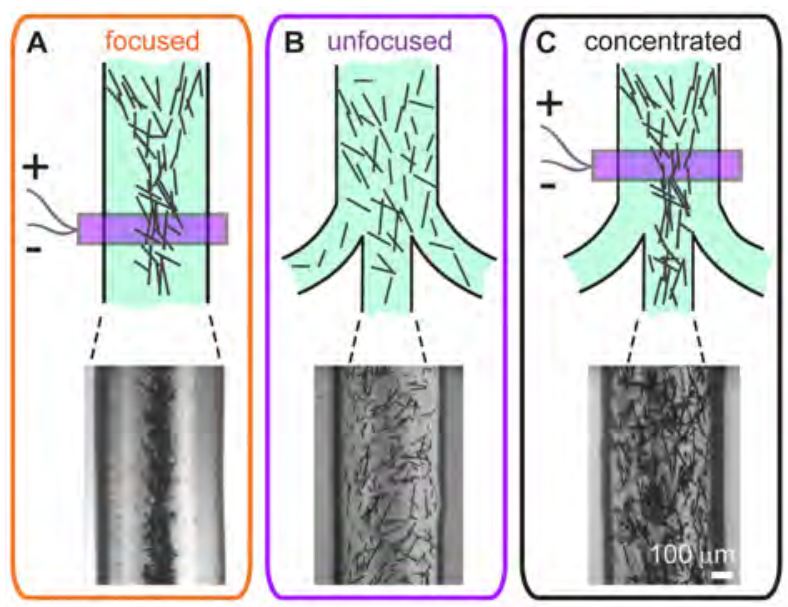

Figure 6: Schematics of channel geometry and control conditions (top) and micrographs of $\mathrm{SiC}$ /epoxy print lines (bottom): (A) focused in a single channel, and in branched channels in the (B) unfocused and (C) single-stage-focused (concentrated) conditions. All micrographs represent $0.8 \mathrm{vol} \%$ nominal $\mathrm{SiC}$ compositions.

cations, the pure matrix sheath surrounding the focus zone would provide insulation required for electronic networks. In other applications, it may be desirable to remove the sheath by employing 'waste' channels downstream of focusing stages, producing print lines with higher volume fraction than the starting ink, in either unfocused (Fig. 5C, 6C) or focused (Fig. 5D) conditions, as discussed further below.

With higher-aspect-ratio particles, clogging of microchannels becomes challenging; acoustic focusing is advantageous in this respect as particles are focused to the center of the channel creating an effective sheath flow. However, each branched channel geometry will present a fundamental clogging limit in which particles will tend to lodge and accumulate at branch points. For example, in the present case, it is possible to print a focused, $1.9 \mathrm{vol} \% \mathrm{SiC}$ fiber ink from an unbranched channel, but this level of particle loading tends to cause clogging. The approximate practical maximum in branched channels with the geometry used in this work is 0.8 vol\%; however, the ability to focus and concentrate in the branched device Fig. 6C leads to output 'concentrated' particle vol\% of approximately 1.3 (estimated from manual measurements of particle area fractions in brightfield micrographs, as described in Materials and Methods). (We note that perfect 'filtration' behavior in the branched device, with $100 \%$ particle focusing efficiency, would lead to an absolute maximum in concentration of $1.6 \mathrm{vol} \%$ for this device geometry). One advantage of acoustic focusing in this arena, however, is its scalability, and by matching excitation frequencies to larger channels in tandem with consideration of transport and focusing-zone parameters[34], it is reasonable to expect that jamming can be mitigated for a given desired particle size and loading. Similarly, one can reasonably expect that optimization of the branch geometry can be exploited to reduce 
A

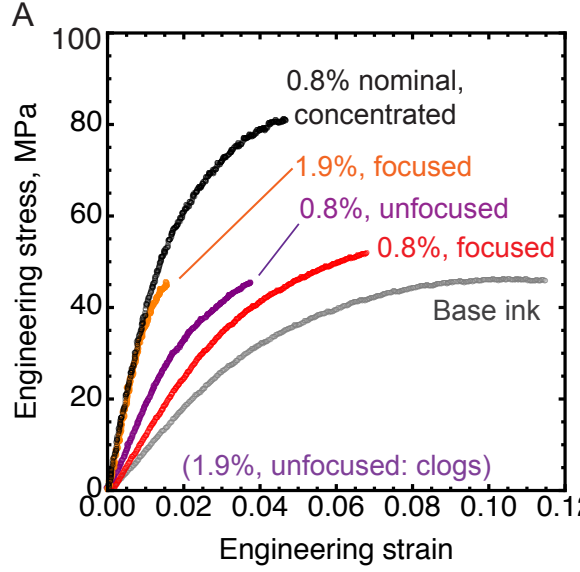

B

\begin{tabular}{|c|c|c|c|c|c|c|c|c|}
\hline \multirow[t]{2}{*}{ Ink Type } & \multirow[t]{2}{*}{ Focusing } & \multicolumn{3}{|c|}{$E(\mathrm{GPa})$} & \multicolumn{3}{|c|}{$\sigma(\mathrm{MPa})$} & \multirow[t]{2}{*}{$n$} \\
\hline & & Ave., St. Dev. & Min. & Max. & Ave., St. Dev. & Min. & Max. & \\
\hline Base & & $1.2 \pm 0.4$ & 0.7 & 1.5 & $43 \pm 8$ & 31 & 52 & 5 \\
\hline $0.8 \% \operatorname{SiC}(A, B)$ & $x$ & $1.4 \pm 0.5$ & 1.0 & 2.2 & $46 \pm 9$ & 32 & 53 & 5 \\
\hline $0.8 \% \operatorname{SiC}(A, B)$ & & $2.3 \pm 0.5$ & 1.9 & 2.9 & $48 \pm 4$ & 42 & 52 & 5 \\
\hline $1.9 \% \mathrm{SiC}(\mathrm{C})$ & $x$ & $3.4 \pm 0.4$ & 3.0 & 3.8 & $45 \pm 8$ & 36 & 53 & 5 \\
\hline $1.9 \% \mathrm{SiC}(\mathrm{C})$ & $(n / a)^{*}$ & & & & & & & \\
\hline $\begin{array}{l}0.8 \% \mathrm{SiC} \\
(2-\text { stage device) }\end{array}$ & First stage & $3.4 \pm 0.3$ & 3.0 & 3.9 & $75 \pm 9$ & 64 & 92 & 7 \\
\hline
\end{tabular}

Figure 7: (A) Mechanical characterization of SiC/epoxy inks for tensile tests of the base ink material (epoxy and filler with no particles) as well as particle-laden inks with either 0.8 or $1.9 \mathrm{vol} \% \mathrm{SiC}$ fibers, for the focusing conditions illustrated in Figure 6. (B) Young's modulus (E) and tensile strength $(\sigma)$ values for printed inks. The mean and standard deviation is reported for at least five samples in each case.

jamming.

The tensile test results in Fig. 7A,B indicate that both particle volume fraction and microstructure affect the modulus and strength of printed parts. As expected, Young's modulus generally increases with increasing particle concentration. However, the different microstructures (focused vs. unfocused) exhibit different moduli for the same particle loading, with only the latter approaching values estimated from an upper-bound rule-of-mixtures calculation[37]. A possible explanation for the lower moduli of focused samples is a difference in stress transfer from particles to the matrix (vs. that in unfocused samples with distributed fibers), as suggested by the shear-lag model of Halpin and Tsai[41] for short-fiber composites. In this model, the composite modulus is a function of a shape parameter that scales inversely with fiber diameter. In the focused condition, it is likely that aggregated fibers are close enough to preclude load transfer across the entire fiber surface: in essence, the surface-area-to-volume-ratio decreases for a focused 'band' of closely arranged fibers, leading to lower composite modulus values for a given particle volume fraction. Thus, depending on the application, it may be desirable to adjust deposition modality for a given part or employ different modes within the same part. For example, the ability to modulate the stiffness locally in a printed part by temporally varying excitation can be exploited for functionally graded materials or custom lattice structures. In terms of overall stiffness and tensile strength, these results are highly promising, as even the small volume percentage of $\mathrm{SiC}$ particles used in the present work have moderate effects on measured Young's modulus (Fig. 7B) and the strength of concentrated samples rivals that of epoxy composites with higher volume fractions of stiffer carbon fibers[4]. 
It is worth noting that while the focus here is on microfluidic print nozzles, the approach is likely also scalable upwards in terms of particle size, in that acoustic focusing forces increase with the volume of the particles in solution. One caveat is that large channels will require higher acoustic energy densities, which can lead to significant heating. (In the current devices, active cooling is used on the print nozzle for the highest excitation voltages to mitigate such effects, and it is reasonable to expect such cooling strategies can also be scaled upwards.) On the other hand, the approach is highly parallelizable in the sense that multiple print nozzles of the scale presented here can be utilized simultaneously.

\section{Conclusion}

We demonstrate acoustic-field-assisted deposition of two-phase composites, including glass and $\mathrm{BaTiO}_{3}$ microspheres as well as $\mathrm{SiC}$ fibers in epoxy, using microfluidic print heads coupled to inexpensive piezoelectric actuators. The microstructure of printed lines were manipulated via acoustic excitation and printing stage parameters. Using classical theories of acoustophoresis and measurements of ink rheology, we identify ink compositions suitable for both deposition and focusing in time-scales relevant to 3D-printing. Two print modalities for acousticallyassisted deposition have been demonstrated: the focusing of particles within a matrix sheath that enables printing of particle volume fractions that otherwise cause jamming, and the use of multiple-stage focusing in branched channels to deliver print lines with higher particle volume fractions than the feed ink. Mechanical characterization of printed materials with varying microstructures show that moderate gains in strength are possible with even modest additions of $\mathrm{SiC}$ fibers in epoxy. As a relatively material agnostic technique for microstructural control, acoustic-focusing-assisted additive manufacturing greatly expands the library of printable multiphase inks and is thus highly complementary to existing and emerging 3D-printing technologies.

\section{Acknowledgments}

R.R.C., T.R.R., R.C.F., J.D.C., and M.R.B. gratefully acknowledge the support of the Institute for Collaborative Biotechnologies through contract no. W911NF-09-D0001 from the U.S. Army Research Office, as well as the use of the MRL Shared Experimental Facilities, supported by the MRSEC Program of the NSF under Award No. DMR 1121053. We also acknowledge use of the Microfluidics Laboratory and the NanoStructures Cleanroom Facility at the California Nanosystems Institute as well as the Nanofabrication Facility (a part of the NSF-funded National Nanotechnology Infrastructure Network) at UCSB. Finally, we thank Kirk Fields at UCSB for assistance with tensile tests.

Author Contributions R.R.C., T.R.R., and M.R.B. designed experiments and wrote the manuscript. R.R.C., T.R.R., R.C.F., and J.D.C. carried out experiments. B.G.C. 
provided guidance on ink development. All authors edited the manuscript.

Competing Interests The authors declare that they have no competing financial interests.

Data and materials availability: Additional data and materials are available online.

[1] N. W. Bartlett, M. T. Tolley, J. T. B. Overvelde, J. C. Weaver, B. Mosadegh, K. Bertoldi, G. M. Whitesides, R. J. Wood, A 3d-printed, functionally graded soft robot powered by combustion, Science 349 (2015) 161-165. doi:10.1126/science.aab0129.

[2] P. Calvert, T. L. Lin, H. Martin, Extrusion freeform fabrication of choppedfibre reinforced composites, High Perform. Polym. 9 (1997) 449-456. doi:10.1088/0954-0083/9/4/008.

[3] J. Peng, T. L. Lin, P. Calvert, Orientation effects in freeformed short-fiber composites, Compos. Part A-Appl. Sci. 30 (2) (1999) 133-138. doi:10.1016/S1359835X(98)00110-9.

[4] B. G. Compton, J. A. Lewis, 3D-Printing of Lightweight Cellular Composites, Adv. Mater. 26 (2014) 5930-5935.

[5] B. Y. Ahn, E. B. Duoss, M. J. Motala, X. Guo, S.-I. Park, Y. Xiong, J. Yoon, R. G. Nuzzo, J. A. Rogers, J. A. Lewis, Omnidirectional printing of flexible, stretchable, and spanning silver microelectrodes, Science 323 (2009) 15901593. doi:10.1126/science.1168375.

[6] B. Derby, Inkjet printing of functional and structural materials: Fluid property requirements, feature stability, and resolution, Annu. Rev. Mater. Res. 40 (2010) 395-414. doi:10.1146/annurev-matsci-070909-104502.

[7] M. S. Onses, E. Sutanto, P. M. Ferreira, A. G. Alleyne, J. A. Rogers, Mechanisms, capabilities, and applications of high-resolution electrohydrodynamic jet printing, Small 11 (2015) 4237-4266. doi:10.1002/smll.201500593.

[8] S. Korkut, D. A. Saville, I. A. Aksay, Collodial cluster arrays by electrohydrodynamic printing, Langmuir 24 (2008) 12196-12201. doi:10.1021/la8023327.

[9] H. Lee, B. Seong, J. Kim, Y. Jang, D. Byun, Direct alignment and patterning of silver nanowires by electrohydrodynamic jet printing, Small 10 (2014) 39183922. doi:10.1002/smll.201400936.

[10] T. Ahn, H.-J. Kim, J. Lee, D.-G. Choi, J.-Y. Jung, J.-H. Choi, S. Jeon, J.-D. Kim, J.-H. Jeong, A facile patterning of silver nanowires using a magnetic printing method, Nanotechnology 26 (34) (2015) 345301. doi:10.1088/0957$4484 / 26 / 34 / 345301$. 
[11] L. Wang, F. Li, M. Kuang, M. Gao, J. Wang, Y. Huang, L. Jiang, Y. Song, Interface manipulation for printing three-dimensional microstructures under magnetic guiding, Small 11 (2015) 1900-1904. doi:10.1002/smll.201403355.

[12] E. Sutanto, K. Shigeta, Y. K. Kim, P. G. Graf, D. J. Hoelzle, K. L. Barton, A. G. Alleyne, P. M. Ferreira, J. A. Rogers, A multimaterial electrohydrodynamic jet (e-jet) printing system, J. Micromech. Microeng. 22 (2012) 045008. doi:10.1088/0960-1317/22/4/045008.

[13] G. H. Kim, D. K. Moeller, Y. M. Shkel, Orthotropic polymeric composites with microstructure tailored by electric field, J. Compos. Mater. 38 (2004) 18951909. doi:10.1177/0021998304048415.

[14] J. Holmes, Larry R., J. C. Riddick, Research summary of an additive manufacturing technology for the fabrication of $3 \mathrm{~d}$ composites with tailored internal structure, JOM 66 (2014) 270-274. doi:10.1007/s11837-013-0828-4.

[15] H. L. Tekinalp, V. Kunc, G. M. Velez-Garcia, C. E. Duty, L. J. Love, A. K. Naskar, C. A. Blue, S. Ozcan, Highly oriented carbon fiber-polymer composites via additive manufacturing, Compos. Sci. Technol. 105 (2014) 144-150. doi:10.1016/j.compscitech.2014.10.009.

[16] R. J. Crowson, M. J. Folkes, P. F. Bright, Rheology of short glass fiberreinforced thermoplastics and its application to injection molding i. fiber motion and viscosity measurement, Polym. Eng. Sci. 20 (1980) 925-933. doi:10.1002/pen.760201403.

[17] A. Lenshof, C. Magnusson, T. Laurell, Acoustofluidics 8: Applications of acoustophoresis in continuous flow microsystems, Lab Chip 12 (2012) 12101223. doi:10.1039/C2LC21256K.

[18] A. H. J. Yang, H. T. Soh, Acoustophoretic sorting of viable mammalian cells in a microfluidic device, Anal. Chem. 84 (2012) 10756-10762. doi:10.1021/ac3026674.

[19] T. Laurell, F. Petersson, A. Nilsson, Chip integrated strategies for acoustic separation and manipulation of cells and particles, Chem. Soc. Rev. 36 (2007) 492506. doi:10.1039/b601326k.

[20] J. K. Lim, D. C. J. Chieh, S. A. Jalak, P. Y. Toh, N. H. M. Yasin, B. W. Ng, A. L. Ahmad, Rapid magnetophoretic separation of microalgae, Small 8 (2012) 1683-1692. doi:10.1002/smll.201102400.

[21] F. Xu, T. D. Finley, M. Turkaydin, Y. Sung, U. A. Gurkan, A. S. Yavuz, R. O. Guldiken, U. Demirci, The assembly of cell-encapsulating microscale hydrogels using acoustic waves, Biomaterials 32 (2011) 7847-7855. doi:10.1016/j.biomaterials.2011.07.010. 
[22] P. Calvert, Printing cells, Science 318 (2007) 208-209. doi:10.1126/science.1144212.

[23] M. Saito, T. Daian, K. Hayashi, S.-y. Izumida, Fabrication of a polymer composite with periodic structure by the use of ultrasonic waves, J. Appl. Phys. 83 (1998) 3490-3494. doi:http://dx.doi.org/10.1063/1.366561.

[24] M. Saito, K. Itagaki, K. Hayashi, K. Tsubata, Composite materials with ultrasonically induced layer or lattice structure, Jpn. J. Appl. Phys. 38 (1999) 3028-3031. doi:10.1143/JJAP.38.3028.

[25] M. Saito, Y. Imanishi, Host-guest composites containing ultrasonically arranged particles, J. Mater. Sci. 35 (2000) 2373-2377. doi:10.1023/A:1004745927648.

[26] M. D. Haslam, B. Raeymaekers, Aligning carbon nanotubes using bulk acoustic waves to reinforce polymer composites, Compos. Part B-Eng 60 (2014) 9197.

[27] L. V. King, Acoustic radiation pressure on spheres, P. Roy. Soc. Lond. A Mat. 147 (1934) 212-240.

[28] W. Nyborg, Radiation pressure on a small rigid sphere, J. Acoust. Soc. Am. 42 (1967) 947-952. doi:10.1121/1.1910702.

[29] W. Nyborg, Theoretical criterion for acoustic aggregation, Ultrasound Med. Biol. 15 (1989) 93-99. doi:10.1016/0301-5629(89)90157-9.

[30] G. ter Haar, S. J. Wyard, Blood cell banding in ultrasonic standing wave fields: a physical analysis., Ultrasound Med. Biol. 4 (1978) 111-123. doi:10.1016/0301-5629(78)90036-4.

[31] M. A. H. Weiser, R. E. Apfel, E. A. Neppiras, Interparticle forces on red-cells in a standing wave field, Acustica 56 (1984) 114-119.

[32] G. Whitworth, W. T. Coakley, Particle column formation in a stationary ultrasonic field, J. Acoust. Soc. Am. 91 (1992) 79-85. doi:10.1121/1.402622.

[33] H. Bruus, Acoustofluidics 7: The acoustic radiation force on small particles, Lab Chip 12 (2012) 1014-1021. doi:10.1039/C2LC21068A.

[34] R. R. Collino, T. R. Ray, C. Meinhart, M. R. Begley, Scaling relationships describing acoustic nozzles for directed assembly of two-phase materials, in preparation. 
[35] R. R. Collino, T. R. Ray, R. C. Fleming, C. H. Sasaki, H. Haj-Hariri, M. R. Begley, Acoustic field controlled patterning and assembly of anisotropic particles, Extreme Mech. Lett. 5 (2015) 37-46. doi:10.1016/j.eml.2015.09.003.

[36] W. S. Rasband, ImageJ, U. S. National Institutes of Health, Bethesda, Maryland, USA, http://imagej.nih.gov/ij/.

[37] K. K. Chawla, Composite Materials: Science and Engineering, Springer, 2012.

[38] S. M. Hägsater, T. G. Jensen, H. Bruus, J. P. Kutter, Acoustic resonances in microfluidic chips: full-image micro-piv experiments and numerical simulations, Lab Chip 7 (2007) 1336-1344. doi:10.1039/B704864E.

[39] P. B. Muller, R. Barnkob, M. J. H. Jensen, H. Bruus, A numerical study of microparticle acoustophoresis driven by acoustic radiation forces and streaming-induced drag forces, Lab Chip 12 (2012) 4617-4627. doi:10.1039/C2LC40612H.

[40] M. Settnes, H. Bruus, Forces acting on a small particle in an acoustical field in a viscous fluid, Phys. Rev. E 85 (2012) 016327. doi:10.1103/PhysRevE.85.016327.

[41] J. C. Halpin, S. W. Tsai, Effects of environmental factors on composite materials, Tech. rep., AFML-TR-67-423 (1969). 